\title{
Validation of a Method for Accurate and Highly Reproducible Quantification of Brain Dopamine Transporter SPECT Studies
}

Peter S. Jensen ${ }^{1}$, Morten Ziebell ${ }^{1}$, Glenna Skouboe ${ }^{1}$, Usman Khalid ${ }^{1}$, Robin de Nijs ${ }^{2}$, Gerda Thomsen ${ }^{1}$, Gitte M. Knudsen ${ }^{1}$, and Claus Svarer ${ }^{1}$

${ }^{I}$ Neurobiology Research Unit, Rigshospitalet, Copenhagen University Hospital, Copenhagen, Denmark; and ${ }^{2}$ Department of Clinical Physiology, Nuclear Medicine and PET, Rigshospitalet, Copenhagen University Hospital, Copenhagen, Denmark

In nuclear medicine brain imaging, it is important to delineate regions of interest (ROIs) so that the outcome is both accurate and reproducible. The purpose of this study was to validate a new time-saving algorithm (DATquan) for accurate and reproducible quantification of the striatal dopamine transporter (DAT) with appropriate radioligands and SPECT and without the need for structural brain scanning. Methods: In a reconstructed DAT SPECT image, DATquan automatically calculated the ratio at steady state of specifically bound radioligand to nondisplaceable radioligand in tissue $\left(\mathrm{BP}_{\mathrm{ND}}\right)$ within striatal $\mathrm{RO}$ ls that were delineated by use of a semiautomatic template-based alignment approach. DATquan was tested with ${ }^{123} \mathrm{I}-\mathrm{N}$-(3-iodoprop2E-enyl)-2- $\beta$-carbomethoxy-3 $\beta$-(4-methylphenyl) SPECT images from 15 patients. In each image, ROIs were first manually delineated, and then corresponding $\mathrm{BP}_{\mathrm{ND}}$ values were derived by an experienced physician. Afterward, 2 independent novice operators used DATquan to analyze the same 15 images. The resulting DATquan-derived $\mathrm{BP}_{\mathrm{ND}}$ data were compared with the data retrieved by manual delineation to assess the accuracy and reproducibility of DATquan. Also, the operational aspects of DATquan were assessed on the basis of measurements of the mean running time of the algorithm as well as on the basis of quantification of the overlap of the DATquandelineated ROls obtained by the 2 operators. Results: The mean algorithm running time was $3 \mathrm{~min}$, and the operators' striatal ROls had a mean overlap of more than $82 \%$. DATquanderived $\mathrm{BP}_{\mathrm{ND}}$ values obtained by the 2 operators showed high agreement (the mean difference was 0.00 [SD, 0.05] in the striatum, 0.02 [SD, 0.26] in the putamen, and 0.03 [SD, 0.43] in the caudate nucleus). The interoperator variability was $2.2 \%$ (SD, $1.3 \%)$ in the striatum, $11.7 \%$ (SD, 9.9\%) in the putamen, and $12.9 \%(S D, 4.0 \%)$ in the caudate nucleus. DATquan-derived $\mathrm{BP}_{\mathrm{ND}}$ values showed high agreement with the values manually derived by the experienced delineator. Conclusion: DATquan is a freely available, accurate, and highly reproducible method for quantification of DAT binding in the brain by SPECT. Once

\footnotetext{
Received Mar. 16, 2011; revision accepted Jul. 26, 2011.

For correspondence or reprints contact: Claus Svarer, Neurobiology Research Unit, Rigshospitalet, N9201, 9 Blegdamsvej, Copenhagen, DK2100 Denmark.

E-mail: cs@nru.dk

Published online Nov. 9, 2011

COPYRIGHT @ 2011 by the Society of Nuclear Medicine, Inc.
}

implemented in clinics, DATquan will serve as a useful and time-saving tool.

Key Words: SPECT; dopamine transporter; brain imaging; quantification; automatic ROI delineation

J Nucl Med Technol 2011; 39:271-278

DOI: 10.2967/jnmt.111.090324

\section{1 he main pathophysiologic feature of Parkinson disease} is degeneration of dopaminergic neurons in the substantia nigra $(1,2)$. The dopamine transporter (DAT) is a presynaptic protein that can be used as a biomarker to assess the degree of nigrostriatal neurodegeneration (3-8). Specific striatal uptake of SPECT radioligands for the DAT, such as ${ }^{123} \mathrm{I}-N$-(3-iodoprop-2E-enyl)-2- $\beta$-carbomethoxy-3 $\beta$-(4methylphenyl) ( $\left.{ }^{123} \mathrm{I}-\mathrm{PE} 2 \mathrm{I}\right)$, has proven to be a good marker for presynaptic neuronal DAT density. Hence, DAT imaging with SPECT can be used in patients with clinical signs of parkinsonism, particularly those with discrete symptoms and a less obvious diagnosis $(9,10)$.

With SPECT, DAT binding is usually quantified as the ratio of specific binding in striatal regions to nonspecific binding in a reference region devoid of DAT. Without information about brain structure from, for example, cerebral MRI, striatal regions of interest (ROIs) are traditionally manually delineated directly on high-count striatal slices in the reconstructed SPECT image. As recently demonstrated (11), however, such manual, non-MRI-guided techniques are operator dependent and, hence, are often associated with considerable intraoperator variability. For example, Ziebell et al. (11) found mean intraoperator variability in $\mathrm{BP}_{\mathrm{ND}}$ estimates from manually delineated striatal, putamen, and caudate nucleus ROIs of $7.0 \%$ (SD, 4.1\%), 9.7\% (SD, 5.4\%), and $10.2 \%$ (SD, 9.2\%), respectively. These data clearly demonstrate that manual ROI delineation is a particularly critical step in the DAT quantification procedure and that it may, in fact, lead to suboptimal reproducibility of the quantified $\mathrm{BP}_{\mathrm{ND}}$ results. 
To overcome the shortcomings of the manual, operatordependent techniques, several automatic or semiautomatic ROI delineation methods for the quantification of DAT images in a more accurate and reproducible way recently were proposed (12-16). Most of these methods are dependent on the use of the commercially available Brain Analysis Software (Hermes Medical Solutions); hence, their usefulness is limited to SPECT centers working with that specific platform.

In this study, we propose and evaluate an algorithm (called DATquan) that offers fast, accurate, and highly reproducible MRI- and operator-independent quantification of brain DAT SPECT images. DATquan is freely available (it can be downloaded from http://nru.dk/downloads/software/) and is not dependent on any specific commercially available software platform, such as the commercially available Brain Analysis Software, other than the widespread MATLAB environment (The Mathworks, Inc.).

\section{MATERIALS AND METHODS}

\section{Test Subjects}

To validate the performance of the DATquan algorithm in a typical clinical setting, we included a mixed sample of patients who had undergone a diagnostic ${ }^{123}$ I-PE2I SPECT scan at the SPECT laboratory of the Neurobiology Research Unit, Rigshospitalet, Copenhagen University Hospital, Copenhagen, Denmark. We selected 15 patients with different neurologic diseases from an in-house database containing all patients who, since 2003 , have been referred for a ${ }^{123} \mathrm{I}-\mathrm{PE} 2 \mathrm{I}$ SPECT scan. All $\mathrm{BP}_{\mathrm{ND}}$ values reported in the database were based on ROIs manually delineated by an experienced physician, and the selection of the patients was done so as to include a wide spectrum of these manually derived $\mathrm{BP}_{\mathrm{ND}}$ values. The selected sample consisted of 10 men and 5 women who were $66.7 \mathrm{y}$ old (SD, $8.9 \mathrm{y}$; range, 53-84 y) and had a mean striatal $\mathrm{BP}_{\mathrm{ND}}$ value of 2.27 (SD, 1.14; range, 0.59-4.41).

\section{Radioligand Administration}

For each subject, a bolus-infusion protocol was used for the administration of ${ }^{123} \mathrm{I}$-PE2I; the bolus was equivalent to $2.7 \mathrm{~h}$ of infusion (the bolus-to-infusion ratio) (17). The administration of an average intravenous bolus of 75.2 $\mathrm{MBq}$ (range, 68.5-81.8 MBq) of ${ }^{123}$ I-PE2I was immediately followed by a constant infusion (mean, 101.3 MBq; range, $89.5-110.6 \mathrm{MBq}$ ) of ${ }^{123} \mathrm{I}-\mathrm{PE} 2 \mathrm{I}$ for a total of $3 \mathrm{~h}$. During the last hour, the subject was scanned by SPECT.

\section{SPECT Acquisition and Image Reconstruction}

${ }^{123}$ I-PE2I SPECT images were obtained with a triplehead IRIX camera (Philips Medical) fitted with low-energy, all-purpose, parallel-hole collimators (spatial resolution, $8.5 \mathrm{~mm}$ in full width at half maximum at a distance of 10 $\mathrm{cm})$. The mean radius of rotation was $14.8 \mathrm{~cm}$. Each head covered $120^{\circ}$ of the circular orbit. Scans were obtained and stored at fixed angles (continuous mode) with an angular interval of $3^{\circ}$. Six SPECT acquisitions, each with a duration of $10 \mathrm{~min}$, were obtained between 120 and 180 min after ${ }^{123}$ I-PE2I injection. Reconstruction of the images was performed with a MATLAB-based program in $128 \times 128$ matrices (2.33-mm pixels and identical slice thicknesses) by use of standard filtered backprojection with a fourth-order low-pass Butterworth filter at 0.3 Nyquist $\left(0.64 \mathrm{~cm}^{-1}\right)$. The imaging energy window was positioned at $143-175 \mathrm{keV}$. High-energy photons of ${ }^{123}$ I penetrated through the lead of the collimator, and Compton scatter in the scintillation crystal caused erroneous counts in the imaging energy window. A second energy window, positioned at $184-216 \mathrm{keV}$, was used to correct for the down-scattered photons in the imaging window (18). Before reconstruction, the projection images of the second energy window were subtracted from those of the first energy window with a weight of 1.1 (19).

\section{Construction of ROI Template}

An ROI template for DATquan was constructed by averaging mutually normalized reconstructed ${ }^{123}$ I-PE2I SPECT images from 10 healthy volunteers (20). The mutual normalization procedure was based on the "reconcile" routine in the AIR software package (21). The mean age of the volunteers was $53.3 \mathrm{y}$ ( $\mathrm{SD}, 12.7 \mathrm{y}$; range, 25-70 y), and 6 of them were men. The average image consisted of 64 slices with $2.33-\mathrm{mm}$ cubic voxels. A physician with more than $5 \mathrm{y}$ of DAT SPECT experience manually delineated 7 template ROIs (right and left striatum, putamen, and caudate nucleus and a cerebellum reference region) with guidance from the neuroanatomic atlas described by Kretschmann and Weinrich (22). The direct adaptation of ROI coordinates from the atlas required a reorientation of the average template image to the canthomeatally oriented plane. As shown in Figure 1, the manually defined striatal ROIs and the reference ROI each covered 9 consecutive slices in the template. The exact position and configuration of the 7 ROIs are shown in Figure 2. All volunteers gave informed written consent, and the study was performed in accordance with the ethical standards of the Declaration of Helsinki and was approved by the ethics committees of Copenhagen and Frederiksberg (H-B-2008-024).

\section{DATquan Algorithm}

The algorithm was written in MATLAB R2010a (The Mathworks, Inc.). It was constructed to run on a Linux platform, but is not dependent on that. In brief, the algorithm works by normalizing the patient's reconstructed SPECT image to the well-defined template on which anatomically shaped ROIs are available. After these ROIs are transferred to the patient's template-normalized image, the algorithm automatically calculates ROI-specific $\mathrm{BP}_{\mathrm{ND}}$ estimates for the patient. These are eventually compared with a normal database, and a graphical presentation of the comparison is created.

In detail, the DATquan algorithm works in the following way.

Normalization of Patient's Image to ROI Template. First, the patient's reconstructed image undergoes automatic nor- 

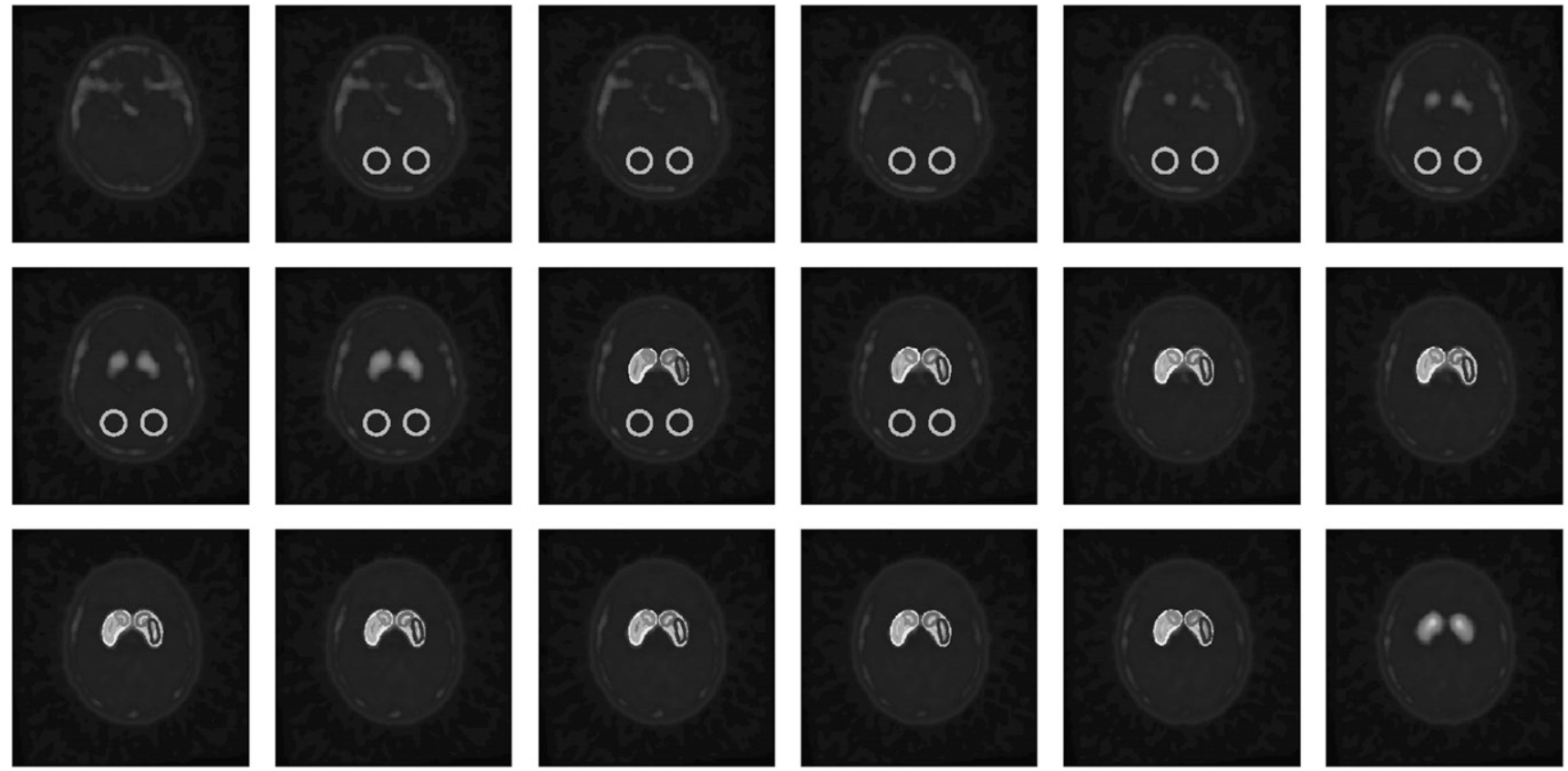

FIGURE 1. Horizontal slices of ${ }^{123}$ I-PE2I template with manually delineated striatum (yellow and cyan), putamen (green and blue), caudate nucleus (pink and red), and reference (orange) ROls. (A color version of this figure is available as a supplemental file online at http://tech.snmjournals.org/.)

malization to the well-defined ROI template by use of an optimal 12-parameter affine transformation that is calculated as the mean of 2 transformations estimated on the basis of the AIR (21) and FLIRT (23) routines. The applied AIR routine uses a "least squares with intensity rescaling" cost function, and the applied FLIRT routine uses a "normalized correlation" cost function, which we found to be the best combination. Furthermore, whereas the AIR routine estimates the transformation on the basis of the template and patient images subjected to thresholding (the respective threshold was set at the median value of each image), the FLIRT routine uses the images as they are.

Delineation of ROIs. After the normalization procedure, the 7 template ROIs were automatically transferred to the normalized patient image in the template space (this image consisted of 64 slices with $2.33-\mathrm{mm}$ cubic voxels). The slice with the highest mean counts in the 2 (left and right) striatal ROIs was automatically identified and selected together with its 4 neighboring slices ( 2 above and 2 below). The remaining 4 slices with striatal ROIs were not used further. The transferred reference ROI was automatically reduced to cover only 5 consecutive slices by ensuring that there were always exactly 2 slices without any ROIs between the lowest (most caudal) striatal slice and the highest (most rostral) reference slice, as shown in Figure 3. Thus, the reference region was positioned as close as possible over the cerebellum.

Fine-Tuning of ROI Positions. Next, the operator had the choice of manually fine-tuning the exact position of the automatically delineated ROIs on the basis of a visual inspection. The purpose of the manual ROI fine-tuning is to enable correction for individual variations in striatal anatomy (such as atrophy or enlarged ventricles) that the automatic patient-to-template normalization procedure cannot account for. To minimize the degree of manual intervention, however, the operator can manually fine-tune only the position of the
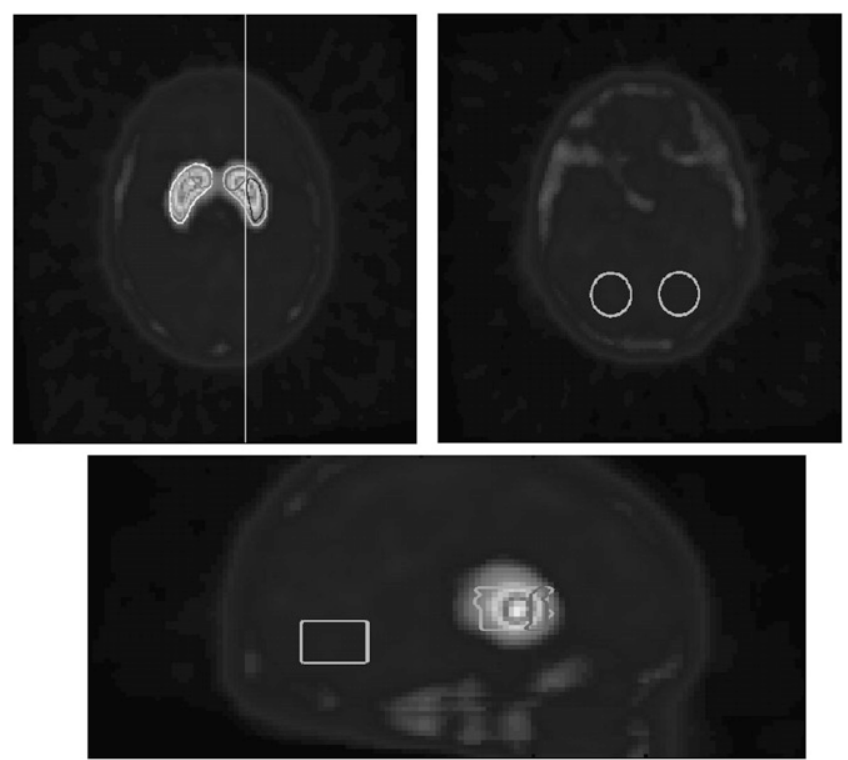

FIGURE 2. Two horizontal slices (upper row) and 1 sagittal slice (lower row) of ${ }^{123}$ I-PE2I template illustrating exact positions and configurations of manually defined striatal and reference ROls. Vertical line in first horizontal slice (upper row, left) illustrates location of sagittal slice. (A color version of this figure is available as a supplemental file online at http://tech. snmjournals.org/.) 

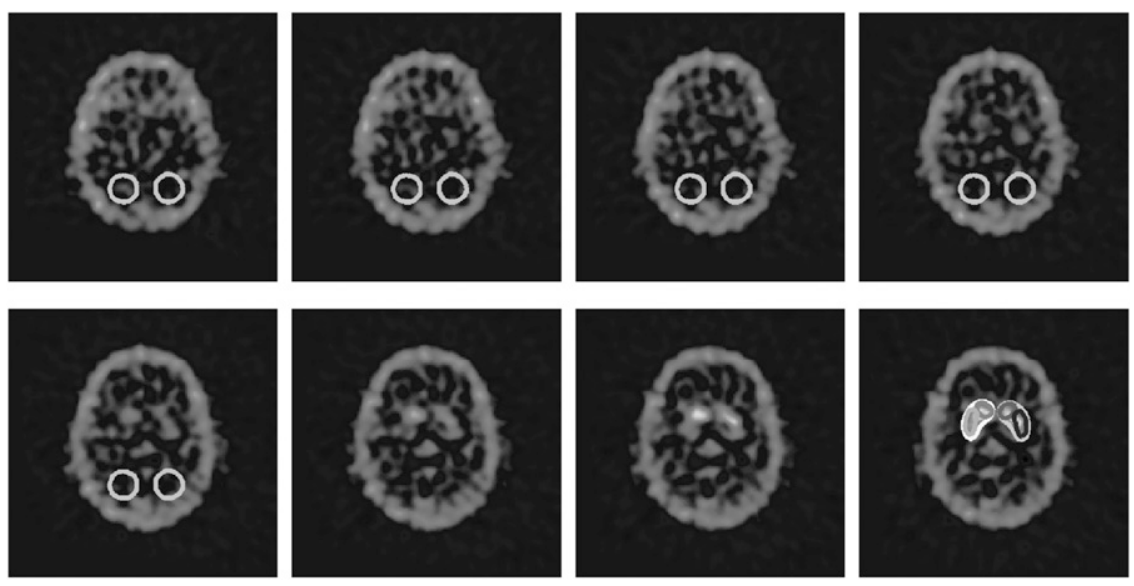

FIGURE 3. Example of part of patient's normalized ${ }^{123}$-PE2I image in template space with automatic DATquan-delineated ROls. Each ROI covers 5 consecutive slices, and there is a gap of 2 slices between reference and striatal ROls. ( $A$ color version of this figure is available as a supplemental file online at http://tech. snmjournals.org/.)
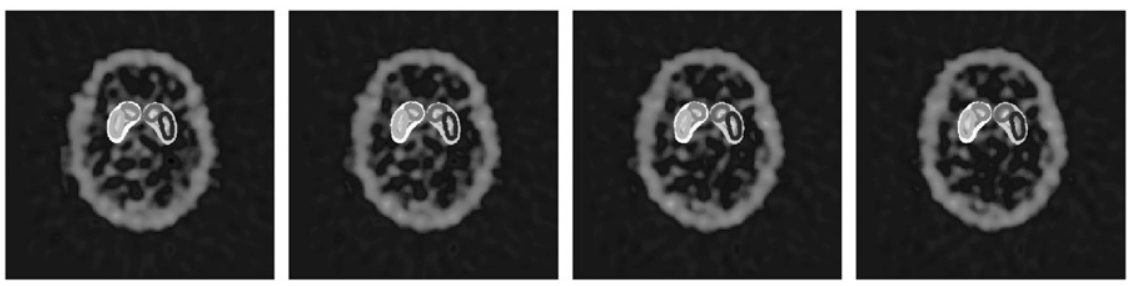

central striatal ROI (on both sides). The operator can apply only in-plane translations to the ROI, and the ROI size is fixed.

The positions of the other ROIs in all 5 consecutive slices were automatically fine-tuned by subjecting them to the same translations as those manually applied to the central striatal ROI.

Calculation of $B P_{N D}$. Eventually, mean counts were automatically extracted from all 7 automatically or semiautomatically delineated ROIs in the normalized image, and ROI-specific equilibrium DAT $\mathrm{BP}_{\mathrm{ND}}$ estimates for the given radioligand were calculated with the following equation:

$$
\mathrm{BP}_{\mathrm{ND}}=\frac{\mathrm{C}_{\mathrm{T}}}{\mathrm{C}_{\mathrm{ND}}}-1 \text {. }
$$

In this equation, $\mathrm{C}_{\mathrm{T}}$ and $\mathrm{C}_{\mathrm{ND}}$ denote mean counts in the tissue ROI and the nondisplaceable reference region, respectively, in accordance with the established consensus nomenclature (24).

\section{DATquan Validation Strategy}

As mentioned earlier, each of the ${ }^{123}$ I-PE2I SPECT images from the selected patients had been quantified in advance by an experienced physician using manual ROI delineation directly on the reconstructed SPECT image. Two independent test operators quantified the reconstructed SPECT images from each of the 15 patients using the DATquan algorithm. Both operators received only one training session (lasting $30 \mathrm{~min}$ ) with DATquan before testing it. On the basis of the resulting 3 sets of quantifications, the accuracy, reproducibility, and usability of DATquan were tested as described here. In all tests, data from all 15 patients were pooled, and right and left ROIs served as separate datasets.
Accuracy. The accuracy of DATquan was assessed by comparing the sets of semiautomatic DATquan-derived $\mathrm{BP}_{\mathrm{ND}}$ estimates from each of the 2 operators with the corresponding manually derived values. The latter were treated as the gold standard. In addition, the accuracy of DATquan was assessed by comparing the volumes (sizes) of the manually delineated and DATquan-delineated ROIs. Each ROI volume was calculated simply as the total number of voxels inside the region times the volume of each $2.33-\mathrm{mm}$ cubic voxel.

Reproducibility. The reproducibility of DATquan was assessed by comparing the sets of DATquan-derived $\mathrm{BP}_{\mathrm{ND}}$ estimates from the 2 operators (referred to as $\mathrm{BP}_{\mathrm{ND}, 1}$ and $\mathrm{BP}_{\mathrm{ND}, 2}$ ) with each other and quantifying the interoperator variability with the following equation:

$$
\text { Interoperator variability }(\%)=\frac{\left|\mathrm{BP}_{\mathrm{ND}, 1}-\mathrm{BP}_{\mathrm{ND}, 2}\right|}{\left(\mathrm{BP}_{\mathrm{ND}, 1}+\mathrm{BP}_{\mathrm{ND}, 2}\right) / 2} \cdot 100 \% \text {. }
$$

Usability. The usability of DATquan was tested on the basis of measurements of the mean running time of the algorithm (on a standard PC with a 2.67-GHz processor and 1 GB of RAM) as well as on measurements of the degree of operator intervention needed, as quantified by the percentage overlap of the 2 operators' manually fine-tuned DATquandelineated ROIs. For each ROI, the percentage overlap was calculated as the number of overlapping voxels divided by the mean number of voxels in the 2 ROI sets.

\section{Statistical Analyses}

For all of the validation tests in which 2 sets of derived $\mathrm{BP}_{\mathrm{ND}}$ estimates were compared, a graphical Bland-Altman analysis was conducted. For assessing the accuracy of DATquan, the mean difference derived from the Bland-Altman plot was used to express the error in DATquan-derived $\mathrm{BP}_{\mathrm{ND}}$ 
estimates. For assessing DATquan reproducibility, the mean difference was used to express agreement between the 2 test operators. For the direct comparison of manually derived and DATquan-derived ROI volumes, a paired Student $t$ test with a 1 -tailed distribution and a significance level of 0.01 was conducted. All statistical analyses were performed with GraphPad Prism v5.03 (GraphPad Software Inc.). Unless otherwise stated, values were expressed as mean (SD).

\section{RESULTS}

\section{DATquan Accuracy}

Figure 4 shows the results from the Bland-Altman analysis of each of the 2 operators' sets of semiautomatic DATquanderived $\mathrm{BP}_{\mathrm{ND}}$ estimates in each of the 3 regions and the corresponding manual $\mathrm{BP}_{\mathrm{ND}}$ values. For both operators, the semiautomatic estimates were found to be slightly higher than the manual ones in all 3 regions, demonstrating a weak tendency of DATquan to overestimate $\mathrm{BP}_{\mathrm{ND}}$ values. For operator 1 , the errors were 0.30 (SD, 0.57), 0.56 (SD, 0.78 ), and 0.29 (SD, 0.82) in the striatum, putamen, and caudate nucleus, respectively. For operator 2, the corresponding errors were 0.30 (SD, 0.58), 0.58 (SD, 0.74), and 0.26 (SD, 0.86), respectively. Except for the fact that for both operators the largest absolute errors were found at the highest $\mathrm{BP}_{\mathrm{ND}}$ values, there was no clear systematic variation in the errors.

Also, for both operators, the band of $95 \%$ limits of agreement was found to be slightly narrower for the striatum than for the putamen and caudate nucleus, demonstrating that the DATquan-derived $\mathrm{BP}_{\mathrm{ND}}$ estimates in the striatum had the lowest variability.

Table 1 summarizes the volumes of the manually delineated and DATquan-delineated ROIs. All 4 DATquan-delineated ROIs were found to be larger than the manual ones. The difference in the ROI volumes was substantial for the striatum but negligible for the putamen and caudate nucleus and for the reference ROI. These results were confirmed by a 1-tailed paired Student $t$ test, which revealed that for both operators, the difference was statistically significant only in the striatum. In addition, no statistically significant volume differences were observed between the 2 sets of DATquandelineated ROIs. Also, the variability in the sizes of the DATquan-delineated ROIs was low and was almost equal for the 2 operators.

\section{DATquan Reproducibility}

Figure 5 shows the results from the Bland-Altman analysis of the 2 operators' sets of semiautomatic DATquan-derived $\mathrm{BP}_{\mathrm{ND}}$ estimates in each of the 3 regions. High agreement between the 2 operators' estimates was evident in all 3 regions. Specifically, the differences were 0.00 (SD, 0.05), 0.02 (SD, 0.26), and 0.03 (SD, 0.43) in the striatum, putamen, and caudate nucleus, respectively. For each ROI separately, there was no systematic variation in the difference.

As expected, the $95 \%$ limits of agreement increased as a function of decreasing ROI size; that is, lower interoperator variability was observed for the striatum than for the putamen and caudate nucleus. Specifically, the interoperator variability was $2.2 \%(\mathrm{SD}, 1.3 \%)$ in the striatum, $11.7 \%$

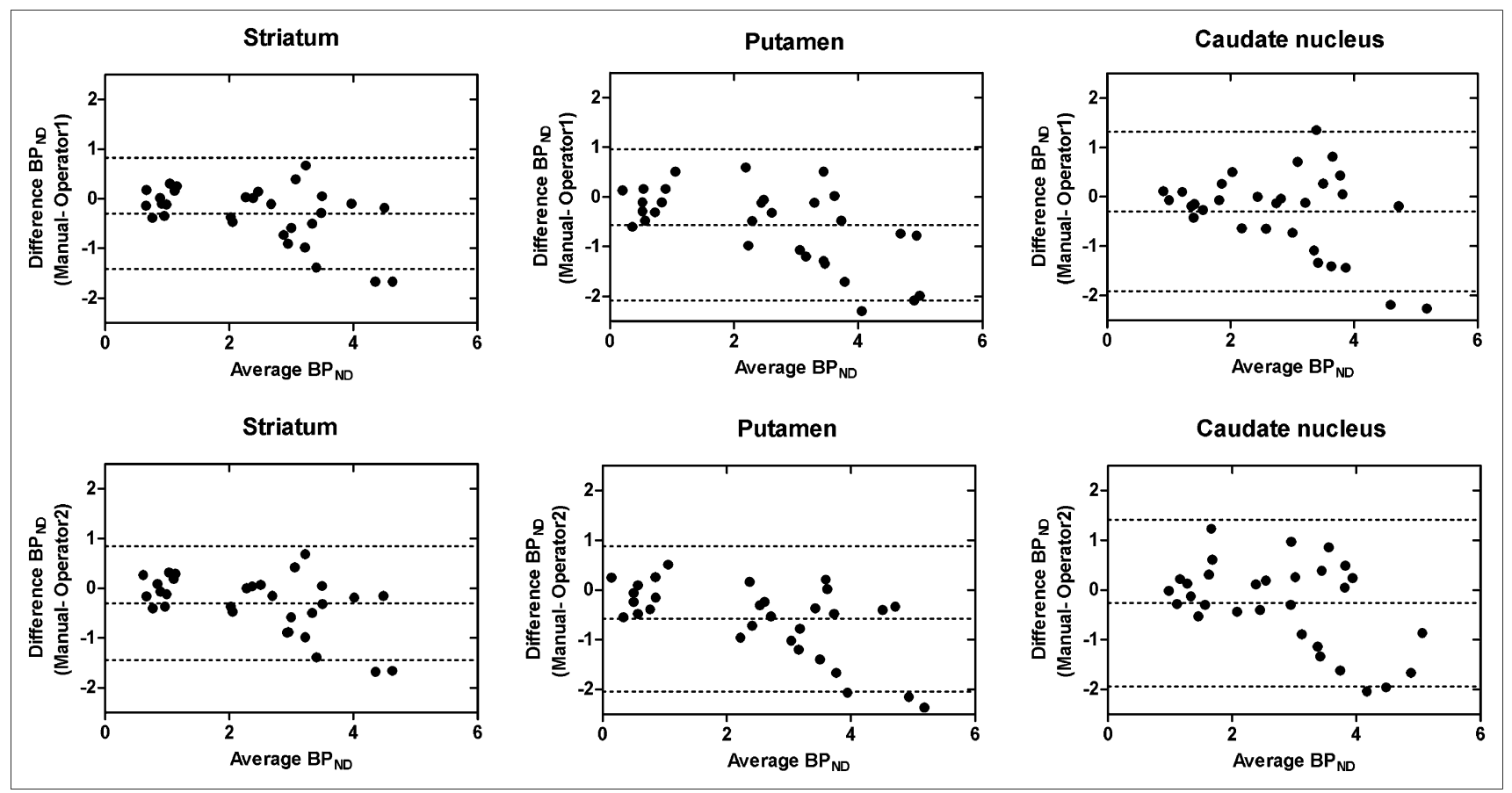

FIGURE 4. Bland-Altman plots of difference between ROI-specific BP $\mathrm{ND}_{\mathrm{ND}}$ estimates obtained manually (by experienced physician) and semiautomatically (with DATquan) against mean of estimates. Top and bottom rows show plots for first and second DATquan operators, respectively. For each ROI-specific plot, mean error and $95 \%$ limits of agreements are indicated by dashed lines. 
TABLE 1

Volumes of Manually and DATquan-Delineated ROIs

\begin{tabular}{|c|c|c|c|}
\hline \multirow[b]{3}{*}{ Area } & \multicolumn{3}{|c|}{ Mean (SD) mL of: } \\
\hline & \multirow{2}{*}{$\begin{array}{c}\text { Manually } \\
\text { delineated ROI }\end{array}$} & \multicolumn{2}{|c|}{$\begin{array}{l}\text { DATquan-delineated } \\
\text { ROI }\end{array}$} \\
\hline & & Operator 1 & Operator 2 \\
\hline Striatum & $6.50(1.95)^{\star}$ & $9.26(0.34)$ & $9.32(0.37)$ \\
\hline Putamen & $2.82(0.80)$ & $2.87(0.22)$ & $2.87(0.25)$ \\
\hline Caudate nucleus & $2.07(0.56)$ & $2.11(0.17)$ & $2.12(0.15)$ \\
\hline Reference & $15.3(4.13)$ & $16.0(2.70)$ & $16.0(2.70)$ \\
\hline
\end{tabular}

${ }^{*}$ Statistically significant $(P<0.01)$ difference between volume of manually delineated $\mathrm{ROI}$ and volume of each DATquan-delineated $\mathrm{ROI}$.

$(\mathrm{SD}, 9.9 \%)$ in the putamen, and $12.9 \%(\mathrm{SD}, 4.0 \%)$ in the caudate nucleus.

\section{DATquan Usability}

By measuring the running time for the use of the algorithm by the 2 operators (time was started when DATquan was initiated and stopped when the output document had been created), we found that both of the operators spent, on average, about 3 min quantifying each of the 15 test studies. For comparison, a manual ROI delineation alone normally takes between 10 and $15 \mathrm{~min}$, depending on how abnormal the DAT image is.

Moreover, on the basis of a comparison of the positions of the 2 sets of semiautomatic DATquan-delineated ROIs for each of the 15 subjects, we found that despite manual fine-tuning of the exact positions of the automatically delineated striatal ROIs, our 2 operators still delineated striatum, putamen, and caudate nucleus ROIs with, on average, $92 \%, 82 \%$, and $85 \%$ overlap.

To investigate the observed tendency of DATquan to overestimate $\mathrm{BP}_{\mathrm{ND}}$ values in more detail, we conducted a Bland-Altman analysis of the region-specific mean counts (i.e., the individual components in the $\mathrm{BP}_{\mathrm{ND}}$ equation) derived from the manually delineated and corresponding DATquan-delineated ROIs. In that analysis, we quantified the percentage error in $\mathrm{C}_{\mathrm{T}}$ as well as in $\mathrm{C}_{\mathrm{ND}}$ to assess whether the overestimation of $\mathrm{BP}_{\mathrm{ND}}$ values was the result of a relative overestimation of $\mathrm{C}_{\mathrm{T}}$ or a relative underestimation of $\mathrm{C}_{\mathrm{ND}}$. Because of the observed high agreement between the 2 operators, Figure 6 shows only the resulting Bland-Altman plots for the first operator.

DATquan-derived estimates of $\mathrm{C}_{\mathrm{T}}$ and $\mathrm{C}_{\mathrm{ND}}$ were found to be lower than the manual ones in all 4 regions, demonstrating a general tendency of DATquan to underestimate region-specific mean counts. Specifically, the percentage errors for $\mathrm{C}_{\mathrm{T}}$ were $17.1 \%$ (SD, 6.5\%), $10.6 \%$ (SD, $16.8 \%$ ), and $17.6 \%$ (SD, 12.9\%) in the striatum, putamen, and caudate nucleus, respectively, whereas the percentage error for $\mathrm{C}_{\mathrm{ND}}$ was $23.9 \%$ (SD, $15.5 \%$ ) in the reference ROI. It is clear that the error was highest in the reference ROI but was statistically significant only in the striatum. Further, for each ROI separately, there was no systematic variation in the error.

As expected, the band of 95\% limits of agreement was found to be narrower for the striatum than for the putamen and caudate nucleus, demonstrating lower variability in the DATquan $\mathrm{C}_{\mathrm{T}}$ (and, in turn, $\mathrm{BP}_{\mathrm{ND}}$ ) estimates in the striatum.

\section{DISCUSSION}

Delineation of ROIs in SPECT images is not easy because the low spatial resolution and the partial-volume effect make the accurate and reproducible delineation of ROIs difficult. Manual delineation is both subjective (high interoperator variability) and time-consuming; hence, faster and more objective methods are needed. This study is one of the first to present a noncommercial and freely available method for accurate and highly reproducible automatic or semiautomatic ROI delineation in DAT SPECT images.

In our DATquan method we used a template-based approach which does not require structural brain information from, for example, a brain MRI of the patient. Having a template-based method that is MRI independent is indeed a strong advantage because many patients at SPECT centers do not routinely undergo a brain MRI scan as part of their investigations and, if they do, the MRI scans are often acquired on different MRI scanners. We also previously demonstrated that MRI-based ROI delineation is not superior
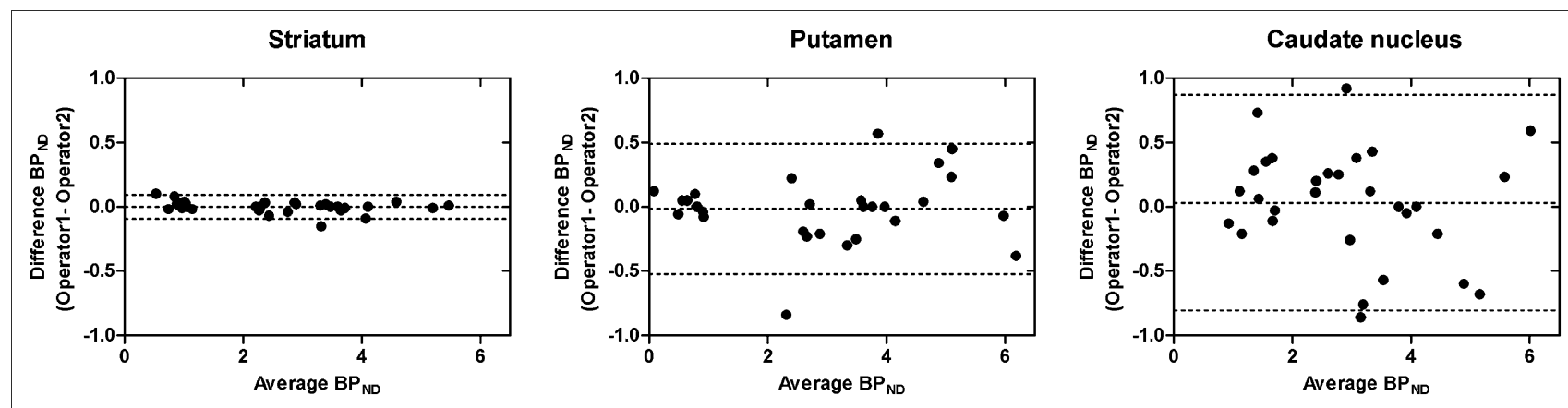

FIGURE 5. Bland-Altman plots of difference between ROI-specific BP $\mathrm{ND}_{\mathrm{N}}$ estimates obtained by 2 DATquan operators against mean of estimates. For each ROI-specific plot, mean difference and $95 \%$ limits of agreements are indicated by dashed lines. 

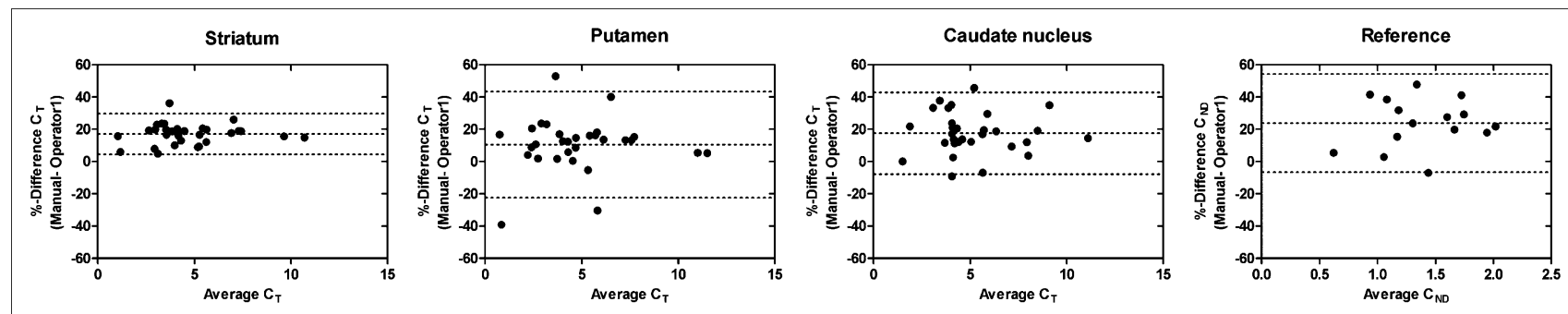

FIGURE 6. Bland-Altman plots of difference between ROI-specific mean counts $\left(\mathrm{C}_{\mathrm{T}}\right.$ and $\left.\mathrm{C}_{\mathrm{ND}}\right)$ derived from manually delineated and DATquan-delineated (operator 1) regions against mean of mean counts. For each ROI-specific plot, mean error and $95 \%$ limits of agreements are indicated by dashed lines.

to manual delineation (11). On the other hand, as described by Calvini et al. (25), there are 2 considerable disadvantages of an automatic ROI delineation method based on a template of healthy individuals. First, such a template is specific for the SPECT camera (acquisition as well as image reconstruction) and, hence, is not directly applicable for other centers. Second, the template is often based on a limited number of healthy individuals and, hence, potentially does not cover the full range of normality. The need for a camera-specific template is, however, only relative as long as the outcome measure is based on $\mathrm{BP}_{\mathrm{ND}}$. In fact, we do not think that these proposed disadvantages are really an issue for the usefulness of our DATquan algorithm. First, we think that all centers should easily be able to make their own camera-specific template as it does not require much effort. Second, the anatomic configuration of a template constructed by averaging brain images from healthy individuals changes only minimally when going from 10 to, for example, 50 individuals; hence, we do not think the exact number of individuals contributing to the template matters that much as well. Instead, we think that correct anatomic positioning of the ROIs in the given template at hand is much more important.

Generally speaking, the expected precision of an automatic nuclear medicine image alignment procedure will depend strongly on the exact tracer distribution profile in the aligned images. Specifically for brain SPECT images, the alignment precision will be better the more distinct high tracer uptake is in cortical regions. Automatic alignment of DAT SPECT images is indeed more difficult than automatic alignment of SPECT flow images (e.g., HMPAO) because of the low cortical uptake. Undoubtedly, a certain fraction of the variability in the $\mathrm{BP}_{\mathrm{ND}}$ estimates comes from inaccuracy in the automatic alignment of the patient images to the template.

Our validation results have shown that the DATquan algorithm produces estimates of $\mathrm{BP}_{\mathrm{ND}}$ values in DAT SPECT images that are in fairly good agreement with corresponding manually derived values. Specifically, a tendency of DATquan to slightly overestimate $\mathrm{BP}_{\mathrm{ND}}$ values (treating the manually derived values as the gold standard) was observed; however, this tendency was not statistically significant in any of the regions. We found that the DATquan- derived $\mathrm{BP}_{\mathrm{ND}}$ overestimation was due to a tendency of DATquan to make a relatively larger underestimation of the mean counts in the reference region $\left(\mathrm{C}_{\mathrm{ND}}\right)$ than of the mean counts $\left(\mathrm{C}_{\mathrm{T}}\right)$ in the striatum, putamen, and caudate nucleus. The observed general underestimation of $\mathrm{C}_{\mathrm{T}}$ and $\mathrm{C}_{\mathrm{ND}}$ was statistically significant only in the striatum, a result that fit well with the observation that only for the striatum were the volumes of the DATquan-delineated ROIs statistically larger than those of the manually delineated ones (increasing the ROI size decreases the regional mean counting rate). We think that for the putamen and caudate nucleus and for the reference ROI, the observed underestimations of the mean counts must have been due to differences in the positioning of the regions or in the exact shapes of the regions.

In fact, we were not surprised to find that our DATquandelineated ROIs were larger than the manually delineated ones. We expected a manual delineator to have a tendency to draw the manual ROIs (excluding the reference ROI) too small by only delineating areas with distinct high activity. Seeing this, we also expected the manually derived $C_{T}$ values to be overestimated, and in turn our DATquandelineated $\mathrm{C}_{\mathrm{T}}$ values to be underestimated (relatively speaking), and clearly this expectation was confirmed by our finding of a general underestimation of $\mathrm{C}_{\mathrm{T}}$ with DATquan.

We have demonstrated that our 2 DATquan test operators presented 2 sets of semiautomatically delineated striatum, putamen, and caudate nucleus ROIs that, on average, were $92 \%, 82 \%$, and $85 \%$ overlapping, respectively. We observed that the variability in the size of the DATquandelineated ROIs was low and almost equal for the 2 operators, and we conclude that DATquan indeed offers a strongly reproducible delineation of the ROIs-a clear advantage of the method.

DATquan-derived $\mathrm{BP}_{\mathrm{ND}}$ estimates obtained by our 2 test operators showed high agreement. The difference between the 2 sets of estimates was 0.00 (SD, 0.05) in the striatum, $0.02(\mathrm{SD}, 0.26)$ in the putamen, and $0.03(\mathrm{SD}, 0.43)$ in the caudate nucleus, leading to an interoperator variability of $2.2 \%(\mathrm{SD}, 1.3 \%)$ in the striatum, $11.7 \%$ (SD, 9.9\%) in the putamen, and $12.9 \%$ (SD, $4.0 \%$ ) in the caudate nucleus.

It is important to stress that no matter how well an automatic method performs, it should always be used as 
a complementary instrument to a visual inspection of the clinical image under analysis. No automatic method is able to integrate all practical knowledge that an experienced physician may have; therefore, an automatic method should never stand alone. An accurate and reproducible automatic method can, nevertheless, be an essential and efficient tool assisting physicians in diagnostic evaluations of most clinical images.

We have shown that 2 independent operators with little training can retrieve highly reproducible $\mathrm{BP}_{\mathrm{ND}}$ estimates in the striatum, putamen, and caudate nucleus in less than 3 min with DATquan. This is a clear advantage of the method, as it reduces time spent in the clinic and, at the same time, eliminates the need for expert personnel specifically trained in the process of manual ROI delineation. For these reasons, we believe that most SPECT centers performing DAT SPECT scans could, in fact, benefit from implementing our freely available algorithm.

Our data analysis was based on the DAT radiotracer ${ }^{123} \mathrm{I}$ PE2I, which is only implemented in a few European SPECT centers, where the use of ${ }^{123}$ I-FP-CIT is much more prevalent. Although this factor may be seen as a limitation of our study, there is no reason to believe that the algorithm would perform differently with other DAT radiotracers as long as a specific template with ROIs for that tracer is available.

\section{CONCLUSION}

We described a freely available algorithm, DATquan, for the automatic quantification of DAT binding in brain DAT SPECT studies. DATquan enables the determination of highly reproducible $\mathrm{BP}_{\mathrm{ND}}$ estimates in striatum, putamen, and caudate nucleus. The interoperator variability in the $\mathrm{BP}_{\mathrm{ND}}$ estimates from the DATquan-delineated ROIs indicates that the semiautomatic $\mathrm{BP}_{\mathrm{ND}}$ estimates in the putamen and caudate nucleus are as reproducible as those obtained with a manual delineation technique, whereas the reproducibility of the estimates in the striatum is higher for DATquan. Further, DATquan-derived $\mathrm{BP}_{\mathrm{ND}}$ estimates agreed well with $\mathrm{BP}_{\mathrm{ND}}$ estimates derived from manually delineated ROIs. Finally, the mean processing time for an operator who has received very limited training in the software is 3 min per brain scan. We conclude that the DATquan algorithm is a useful add-on tool in the everyday SPECT clinic because it produces fast and highly reproducible DAT SPECT quantification results that show good agreement with results obtained by use of a traditional manual ROI delineation technique.

\section{ACKNOWLEDGMENTS}

The Lundbeck Foundation is acknowledged for financial support, the Toyota Foundation is acknowledged for donation of the SPECT scanner, and the Novo Nordisk Foundation is acknowledged for donation of our server systems. No other potential conflict of interest relevant to this article was reported.

\section{REFERENCES}

1. Bernheimer H, Birkmayer W, Hornykiewicz O, Jellinger K, Seitelberger F. Brain dopamine and the syndromes of Parkinson and Huntington: clinical, morphological and neurochemical correlations. J Neurol Sci. 1973;20:415-455.

2. Kaufman MJ, Madras BK. Severe depletion of cocaine recognition sites associated with the dopamine transporter in Parkinson's-diseased striatum. Synapse. 1991;9:43-49.

3. Allard PO, Rinne J, Marcusson JO. Dopamine uptake sites in Parkinson's disease and in dementia of the Alzheimer type. Brain Res. 1994;637:262-266.

4. Bao SY, Wu JC, Luo WF, Fang P, Liu ZL, Tang J. Imaging of dopamine transporters with technetium-99m TRODAT-1 and single photon emission computed tomography. J Neuroimaging. 2000;10:200-203.

5. Brücke T, Asenbaum S, Pirker W, et al. Measurement of the dopaminergic degeneration in Parkinson's disease with $\left[{ }^{123} \mathrm{I}\right]$ beta-CIT and SPECT: correlation with clinical findings and comparison with multiple system atrophy and progressive supranuclear palsy. J Neural Transm Suppl. 1997;50:9-24.

6. Innis RB, Seibyl JP, Scanley BE, et al. Single photon emission computed tomographic imaging demonstrates loss of striatal dopamine transporters in Parkinson disease. Proc Natl Acad Sci USA. 1993;90:11965-11969.

7. Lee CS, Samii A, Sossi V, et al. In vivo positron emission tomographic evidence for compensatory changes in presynaptic dopaminergic nerve terminals in Parkinson's disease. Ann Neurol. 2000;47:493-503.

8. Marshall V, Grosset DG. Role of dopamine transporter imaging in the diagnosis of atypical tremor disorders. Mov Disord. 2003;18(suppl 7):S22-S27.

9. Emond P, Guilloteau D, Chalon S. PE2I: a radiopharmaceutical for in vivo exploration of the dopamine transporter. CNS Neurosci Ther. 2008;14:47-64.

10. Prunier C, Bezard E, Montharu J, et al. Presymptomatic diagnosis of experimental parkinsonism with ${ }^{123}$ I-PE2I SPECT. Neuroimage. 2003;19:810-816.

11. Ziebell M, Pinborg LH, Thomsen G, et al. MRI-guided region-of-interest delineation is comparable to manual delineation in dopamine transporter SPECT quantification in patients: a reproducibility study. J Nucl Med Technol. 2010;38:61-68.

12. Pöpperl G, Radau P, Linke R, Hahn K, Tatsch K. Diagnostic performance of a 3-D automated quantification method of dopamine D2 receptor SPECT studies in the differential diagnosis of parkinsonism. Nucl Med Commun. 2005;26:39-43.

13. Mirzaei S, Zakavi R, Rodrigues M, et al. Fully automated 3D basal ganglia activity measurement in dopamine transporter scintigraphy (Spectalyzer). Ann Nucl Med. 2010;24:295-300.

14. Koch W, Radau PE, Hamann C, Tatsch K. Clinical testing of an optimized software solution for an automated, observer-independent evaluation of dopamine transporter SPECT studies. J Nucl Med. 2005;46:1109-1118.

15. Habraken JB, Booij J, Slomka P, Sokole EB, van Royen EA. Quantification and visualization of defects of the functional dopaminergic system using an automatic algorithm. J Nucl Med. 1999;40:1091-1097.

16. Radau PE, Linke R, Slomka PJ, Tatsch K. Optimization of automated quantification of ${ }^{123}$ I-IBZM uptake in the striatum applied to parkinsonism. J Nucl Med. 2000;41:220-227.

17. Pinborg LH, Ziebell M, Frokjaer VG, et al. Quantification of ${ }^{123} \mathrm{I}$-PE2I binding to dopamine transporter with SPECT after bolus and bolus/infusion. J Nucl Med. 2005;46:1119-1127.

18. Small AD, Prosser J, Motherwell DW, McCurrach GM, Fletcher AM, Martin W. Downscatter correction and choice of collimator in ${ }^{123} \mathrm{I}$ imaging. Phys Med Biol. 2006;51:N307-N311.

19. de Nijs R, Holm S, Thomsen G, Ziebell M, Svarer C. Experimental determination of the weighting factor for the energy window subtraction-based downscatter correction for I-123 in brain SPECT studies. J Med Phys. 2010;35:215-222.

20. Ziebell M, Holm-Hansen S, Thomsen G, et al. Serotonin transporters in dopamine transporter imaging: a head-to-head comparison of dopamine transporter SPECT radioligands ${ }^{123}$ I-FP-CIT and ${ }^{123}$ I-PE2I. J Nucl Med. 2010;51:18851891.

21. Woods RP, Cherry SR, Mazziotta JC. Rapid automated algorithm for aligning and reslicing PET images. J Comput Assist Tomogr. 1992;16:620-633.

22. Kretschmann HJ, Weinrich W. Neuroanatomy and Cranial Computed Tomo graphy. Stuttgart, Germany: Thieme; 1986.

23. Jenkinson M, Smith S. A global optimisation method for robust affine registration of brain images. Med Image Anal. 2001;5:143-156.

24. Innis RB, Cunningham VJ, Delforge J, et al. Consensus nomenclature for in vivo imaging of reversibly binding radioligands. J Cereb Blood Flow Metab. 2007;27: 1533-1539.

25. Calvini P, Rodriguez G, Inguglia F, Mignone A, Guerra UP, Nobili F. The basal ganglia matching tools package for striatal uptake semi-quantification: description and validation. Eur J Nucl Med Mol Imaging. 2007;34:1240-1253. 